\title{
Calcium pyrophosphate dihydrate (CPPD) deposition in ochronotic arthropathy
}

\author{
J McCLURE, PS SMITH, AMANDA A GRAMP
}

From the Division of Tissue Pathology, Institute of Medical and Veterinary Science, Frome Road, Adelaide, South Australia 5000

SUMMARY The post-mortem examination of an unsuspected case of alkaptonuria revealed extensive ochronosis. Histological examination of undecalcified sections of tracheal, costal, femoral and patellar cartilage revealed, in addition to ochronotic pigment, extensive calcium pyrophosphate dihydrate (CPPD) deposition. Similar deposits were present in intervertebral discs and were related to ossification of the discs resulting in partial or complete ankylosis. The calcific deposits were present around chondrocytes in the articular cartilage and this may be an important factor in the initiation of the osteoarthrotic process which characterises ochronotic arthropathy as it affects large diarthrodial joints.

Ochronosis was the name given by Virchow in $1866^{1}$ to a condition characterised by the pigmentation of cartilages, ligaments, tendons and the intima of the large blood vessels of the body. In Virchow's case the pigment was variably light grey, brown or, more usually, black. In histological sections, however, the pigment was everywhere found to be yellow or yellow-brown and for this reason he called the condition ochronosis (ochre $=$ yellow-brown pigment).

Ochronosis is now a recognised consequence of alkaptonuria. This is an inborn error of metabolism, inherited as a Mendelian recessive characteristic, which affects the breakdown of tyrosine and phenylalanine. There is an absence of the enzyme homogentisic acid oxidase in the tissues. Homogentisic acid, therefore, accumulates, appears in the urine and on oxidation turns black. Almost all alkaptonurics become ochronotic by the time they reach middle age ${ }^{2}$ and in about $50 \%$ of these arthropathic changes occur in the cartilages of large diarthrodial joints and in the intervertebral discs. ${ }^{3}$

The morbid anatomical features of ochronosis have been documented by Oppenheimer and Kline ${ }^{4}$ and by Lichtenstein and Kaplan ${ }^{5}$ and in the latter paper there is a good description and illustration of the changes of ochronotic arthropathy as it affects large diarthrodial joints and the vertebral column. In the former there is osteoarthrosis and in the vertebral column there is exostosis with complete or partial disappearance of intervertebral discs and replacement by bone tissue to produce varying degrees of vertebral body fusion.

Accepted for publication 17 March 1983
It has been assumed that the deposition of ochronotic pigment in cartilage alters the characteristics of that material so that osteoarthrotic changes are initiated. The exact mechanisms of this process are, however, mysterious. O'Brien et $a l^{6}$ have suggested that the critical problem in the pathogenesis of the arthropathy is the affinity of cartilage for the pigment or its precursors. More recently Hamilton ${ }^{7}$ has indicated that calcium pyrophosphate dihydrate (CPPD) crystal deposition has been reported in the synovium of peripheral joints in four cases of ochronosis ${ }^{8-11}$ and that calcium hydroxyapatite has been identified in an intervertebral disc of one of these cases. ${ }^{8}$

The present report is about the findings of a post-mortem examination of a previously undiagnosed case of alkaptonuria with extensive ochronosis. In addition to standard histopathological studies a special effort was made to elucidate the nature of observed calcific deposits.

\section{CLINICAL FEATURES}

A 74-year-old man had chronic obstructive airways disease and aortic stenosis. He suffered a recent deterioration in exercise tolerance with dyspnoea at rest. Chest examination revealed bilaterally decreased air entry and fine basal crepitations. Sputum culture grew Pseudomonas aeruginosa. Despite treatment his condition declined inexorably and he died.

He had had pains in the left shoulder and right leg said to be due to arthritis. There was deep brown pigmentation of the sclerae, face and hands which 
was said to have been present since childhood. The clinical diagnosis was of an acral pigmentation syndrome.

\section{NECROPSY FINDINGS}

Post-mortem examination revealed the presence of ochronosis. There was extensive grey-black discolouration of the epiglottic, laryngeal and bronchial cartilages. There was severe calcific aortic stenosis due to grey-black valvular deposits. Urine was taken at necropsy and the presence of homogentisic acid in large amounts was confirmed by mass spectrometry. The chemical screening tests and chromatography were also strongly positive.

The costal cartilages were a uniform deep black colour. An $x$-ray examination of the breast plate showed extensive irregular opacities of the cartilages, ossification of the xiphisternum and ankylosis of the manubriosternal joint (Fig. 1).

There were marked changes in the vertebral column with almost complete loss of intervertebral discs in the thoracic area and complete or partial replacement by bone tissue causing ankylosis of ver-

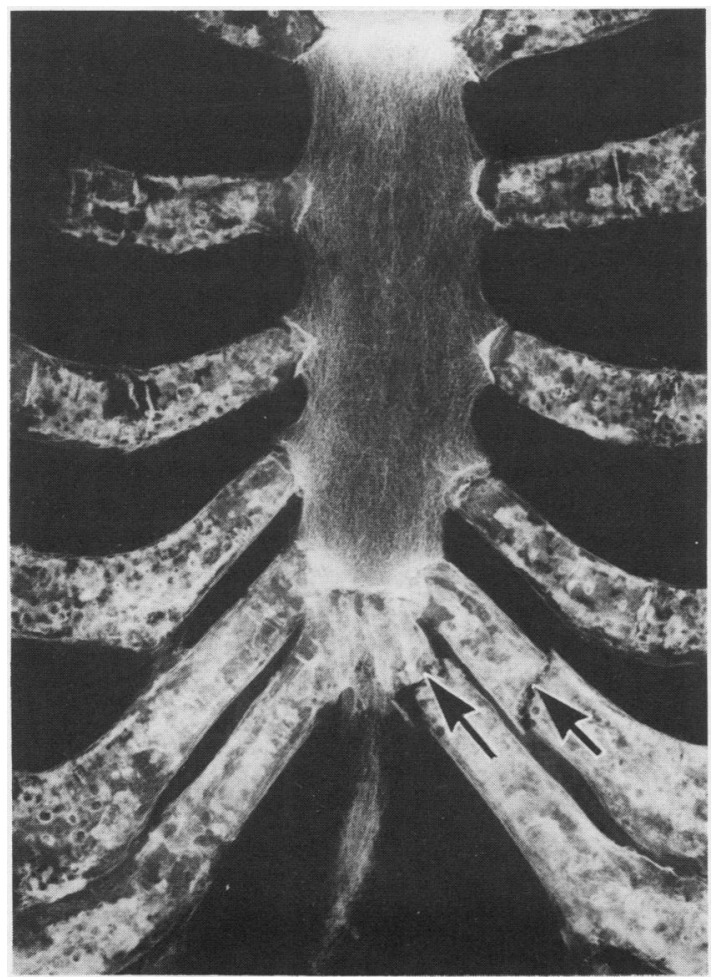

Fig. 1 Contact radiograph of the sternum. Note the radio-opacities of the costal cartilages and the ankylosis of the manubriosternal joint. The breaks (arrowed) in the costal cartilages are artefacts.

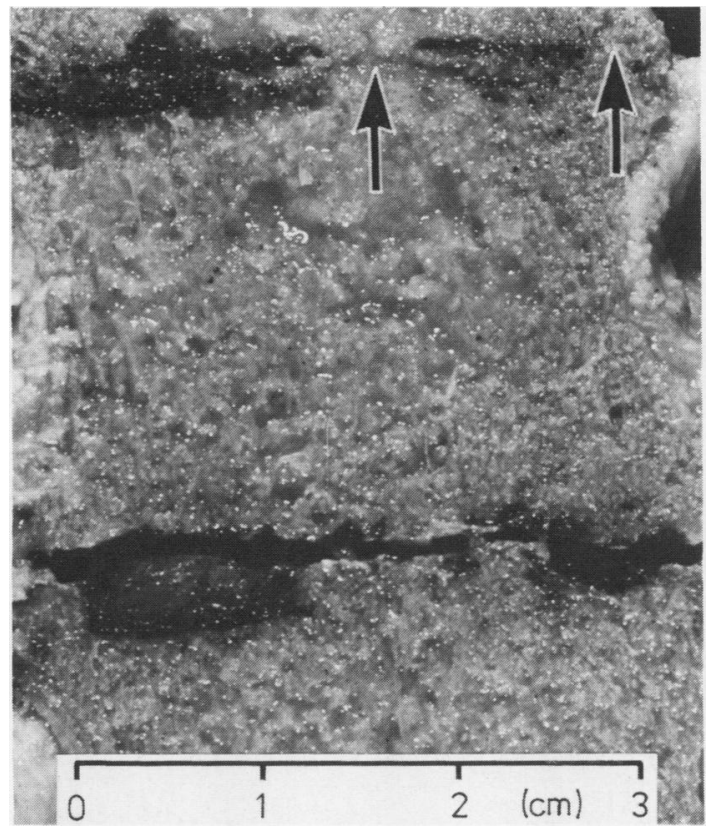

Fig. 2 Partial ankylosis (arrowed) of ochronotic intervertebral discs in the thoracic region.

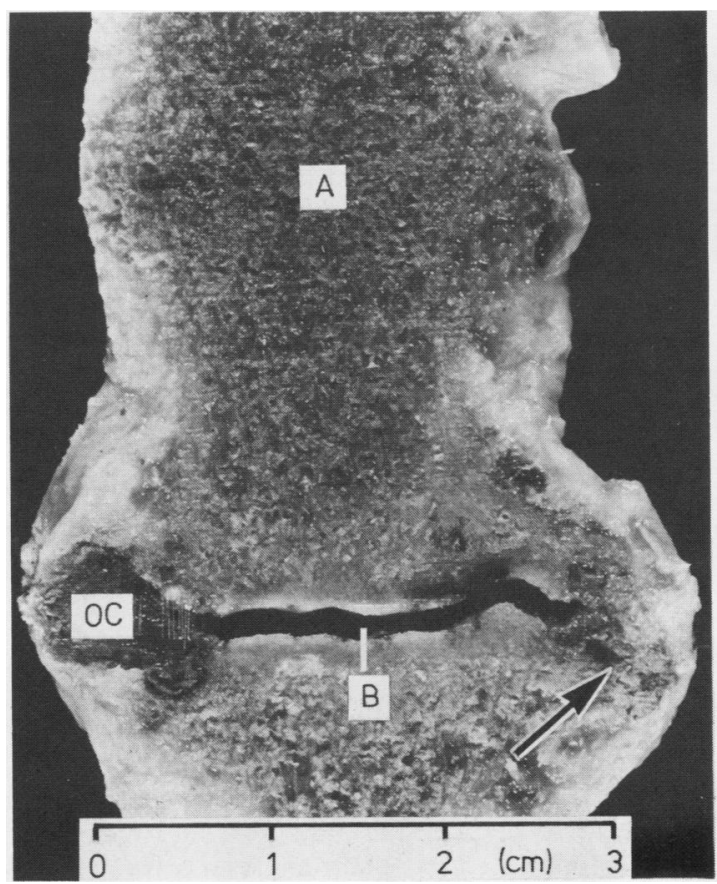

Fig. 3 An ankylosed intervertebral disc is at the level marked $(A)$. The arrow indicates an osteophyte and $(B)$ is an irregular horizontal cleft through the disc site. $O C$ is a residual portion of ochronotic disc material. 
teural bodies. Where there was partial replacement the disc residua were deeply pigmented (Fig. 2). In the thoracolumbar region there was marked irregular osteophytosis. These outgrowths were partly due to bone and partly to superimposed irregular opacities. In some sites of marked osteophyte formation irregular clefts were noted through the horizontal axial plane of the disc and the margins of these clefts were densely sclerotic (Fig. 3).

The articular surface of the head of the left femur was deeply pigmented and generally intact. The right head was distorted with extensive loss of cartilage, eburnation of the subchondral bone and marginal osteophyte formation. The residual cartilage was also deeply pigmented.

In the right knee joint the cartilage of the articular surface of the lower end of the femur was black in colour and completely absent anteriorly. The cartilage of the tibial condyles was an identical colour. Approximately half of the articular cartilage of the patella was absent again with eburnation of subjacent bone. The residual cartilage was a deep black colour.

\section{Material and methods}

Duplicate blocks of tissue were taken from the trachea, the costal cartilages, various sites in the vertebral column, the whole head of the left femur and synovium of the left hip joint, the lower end of the right femur and the right patella. Each block (with the exception of the trachea) included cartilage and related bone. One of each pair was decalcified with $\boldsymbol{x}$-ray control in EDTA, embedded in paraffin wax and sections stained with haematoxylin and eosin (HE). The second of each pair was embedded in Araldite without prior decalcification and sectioned at $7 \mu \mathrm{m}$ with a Jung $\mathrm{K}$ microtome. Multiple sections were cut and stained by a variety of techniques including von Kossa with $\mathrm{HE}$ or van Gieson counterstains, alizarin red S, HE and the morin fluorescent method. Sections were also mounted on glass slides (without removal of Araldite and without staining) for examination by polarising light microscopy.

Portions of the Araldite-embedded left femoral head, costal cartilages and intervertebral discs were cut out of the blocks after the thin sections had been prepared. The cut surfaces of these were polished to within $1 \mu \mathrm{m}$ using diamond paste and coated with carbon for $x$-ray microanalysis by a Jeol 733 superprobe model electron microanalyser with a Kevex 7100 series energy dispersive spectrometer (accelerating voltage $15 \mathrm{kV}$, beam current $9 \mathrm{nA}$, spectrum collection time $20 \mathrm{~s}$, spectrometer take-off angle $=45^{\circ}$ ).

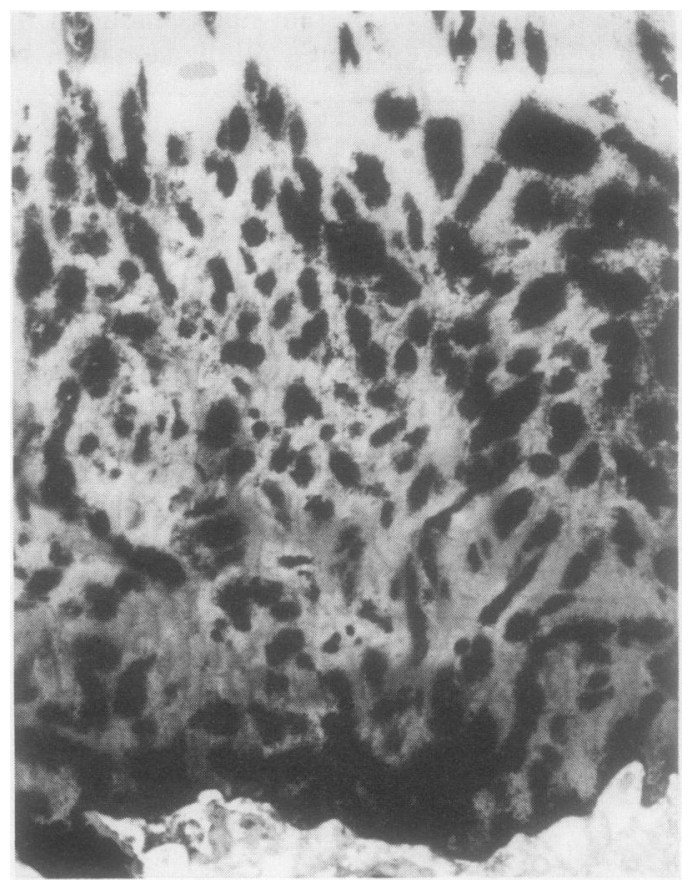

Fig. 4 Calcific deposits (black) around chondrocyte lacunae and in broader, irregular deposition near the costochondral junction. Haematoxylin and eosin and von Kossa stain $\times 40$.

\section{MICROSCOPIC FEATURES}

There was a generalised brown-yellow pigmentation of the matrix of the tracheal cartilaginous rings. Peripherally there were focal positive reactions with the von Kossa technique and alizarin red $S$ indicating the presence of calcium salts. In these zones of reaction the matrix was deeply basophilic on HE staining. The calcium salts were deposited around chondrocyte lacunae and in broader, more irregular plates. The morin stain gave green-white fluorescence at the sites of these deposits and polarising microscopy of unstained sections showed that the deposits exhibited focal weak positive birefringence.

In the HE-stained sections there was a yellowbrown pigmentation of the matrix of the costal cartilage with the brown colouration more marked peripherally. Calcific deposits with staining, fluorescent and polarising properties identical to those described above were seen around chondrocyte lacunae and in broader, irregular, plate-like depositions (Fig. 4). Peripherally there was erosion of both calcified and non-calcified cartilage matrix to produce scalloping and clefting. The erosion was due to multinucleated giant cells behind which there were aggregates of macrophages whose cytoplasm contained fine brown granules (Fig. 5). Beyond these 

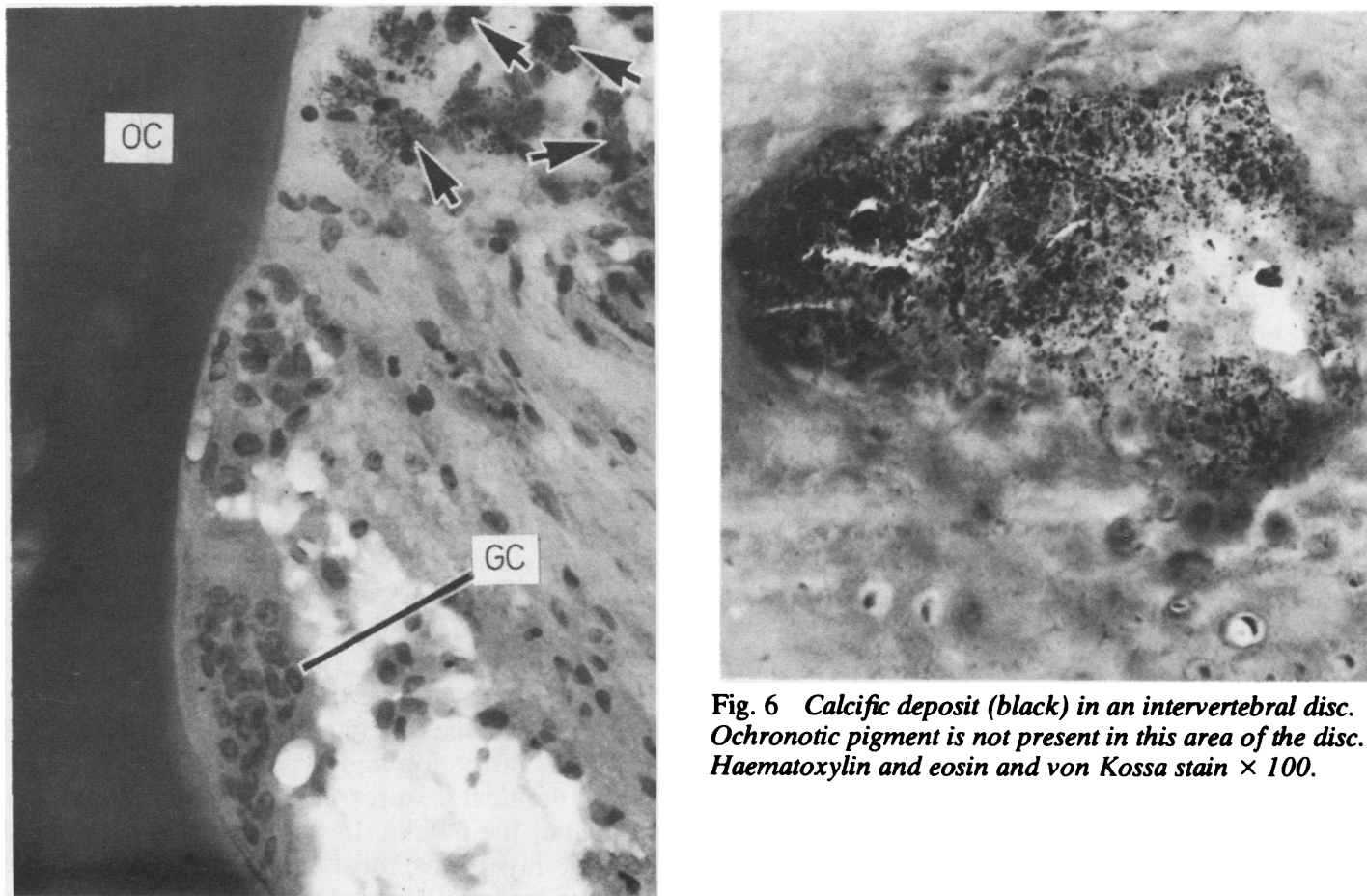

Fig. 6 Calcific deposit (black) in an intervertebral disc. Ochronotic pigment is not present in this area of the disc. Haematoxylin and eosin and von Kossa stain $\times 100$.

Fig. 5 Erosion of non-calcified ochronotic cartilage (OC) by multinucleated giant cells $(G C)$. Macrophages containing granular ochronotic pigment are arrowed. Haematoxylin and eosin and von Kossa stain $\times 200$.

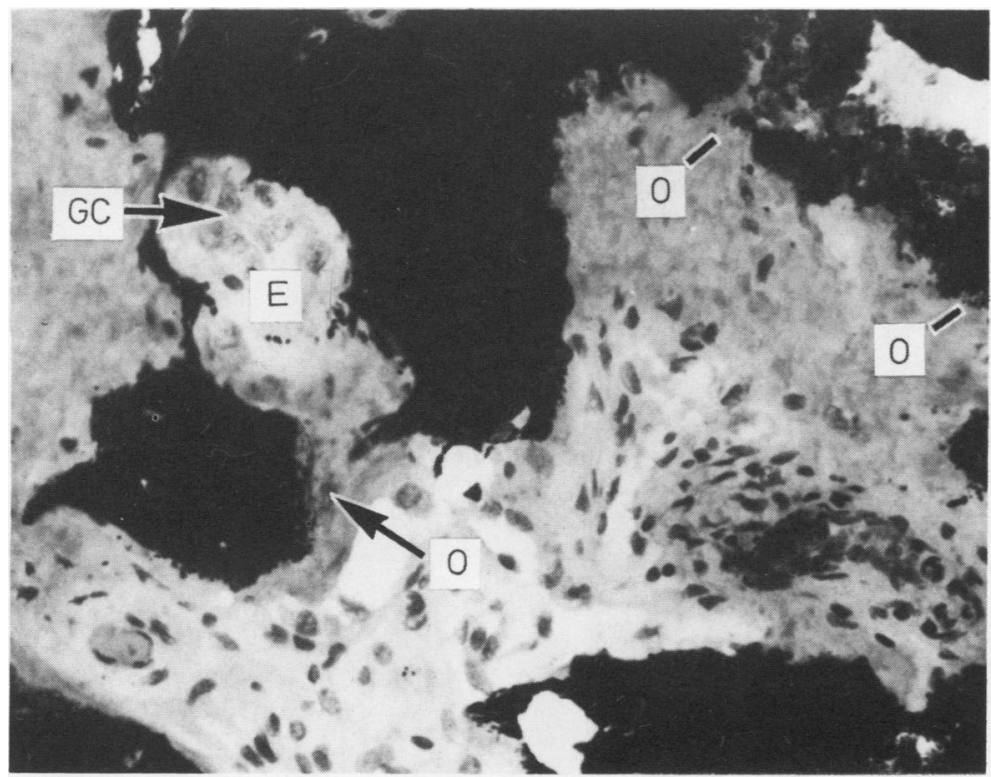

Fig. 7 Excavation (E) of calcific deposit by a multinucleated cell $(G C)$. Osteoid $(O)$ has been laid down on the walls of the proximal part of this excavation. Osteoid $\left(O^{\prime}\right)$ fills other "older" excavations of the calcific deposits. Haematoxylin and eosin and von Kossa stain $\times 100$. 
macrophages active osteoblasts laid down osteoid on the walls of the areas of erosion. This osteoid was undergoing calcification and blended into definitive trabecular bone.

The intervertebral discs were not uniformly pigmented. The pigment was present in numerous discrete zones. Calcific deposits with staining reactions identical to those described above were present as discrete foci randomly distributed throughout both annulus and nucleus of the discs (Fig. 6). The calcific deposits in some areas were closely related to pigmented matrix but in others there was no evident relation. The bone endplates were markedly irregular and through gaps in them highly vascular tissue protruded into the matrix of the discs. The apex of this tissue was composed of multinucleated giant cells. In many zones these cells had eroded into calcific deposits forming excavations upon whose walls osteoblasts had laid down osteoid and mineralised bone in trabecular structures (Fig. 7). These areas of neo-osteogenesis formed bars of bone which extended for varying distances into the discs and almost bridged one vertebral body to another. In some discs there was complete or partial bony ankylosis with foci of residual pigmented matrix in the latter. In some zones small foci of pigmented disc matrix were incorporated into trabecular structures.

At one joint there was a marked exophytic proliferation in relation to large calcific deposits. There was a narrow cleft space between bone surfaces where the disc had been replaced. These surfaces were eburnated and the related bone tissue was hyperplastic. Small calcific deposits and fragments of pigmented disc matrix were occasionally included in this hyperplastic bone (Fig. 8).

The articular cartilage of the head of the femur was pigmented. This was of variable concentrationhighest in the deep and least in the superficial layer. In the intermediate layer calcific deposits were present in the matrix around chondrocyte lacunae and as discrete homogeneous masses (Fig. 9).

The synovium of the hip joint showed a prominent villous pattern with focal synoviocyte hyperplasia but no significant cell hypertrophy or nuclear pleomorphism. There was extensive calcific deposition mainly on the surface of the villous structures (Fig. 10). There was no fibrin deposit or evidence of an acute inflammatory process. Shards of pigmented cartilage were embedded in the stroma and there was a macrophage and macrophage polykaryon reaction to some of these fragments.

The cartilage of the lower end of the femur was fibrillated to a variable degree (superficial to deep). There was a zone of complete loss of articular cartilage with eburnation and hyperplasia of subchondral bone. Identical features were present in the articular surface of the patella. In the preserved cartilage the deepest layer was most pigmented. In the intermediate layer calcific deposits were disposed around chondrocytic lacunae and as discrete masses.

\section{X-RAY MICROANALYSIS}

With the Jeol 733 superprobe the specimens were examined using back scattered electron imaging and several components could be distinguished by the

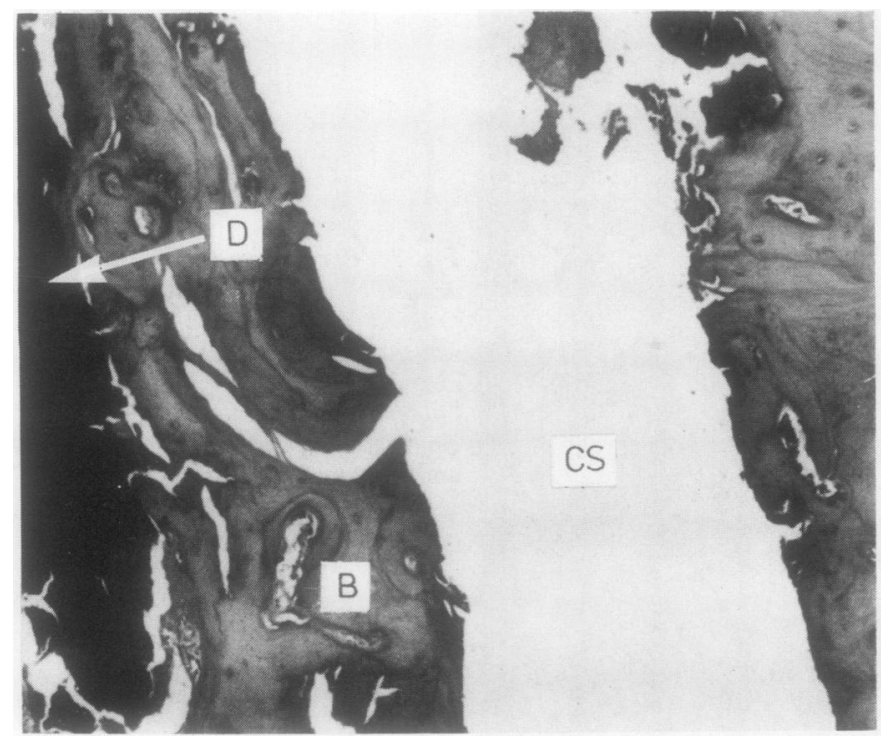

Fig. 8 Residual ochronotic disc material (D) embedded in hyperplastic bone $(B)$ which lines a cleft space (CS) at the site of the intervertebral disc (B) shown in Fig. 3. Haematoxylin and eosin and von Kossa stain $\times 40$. 


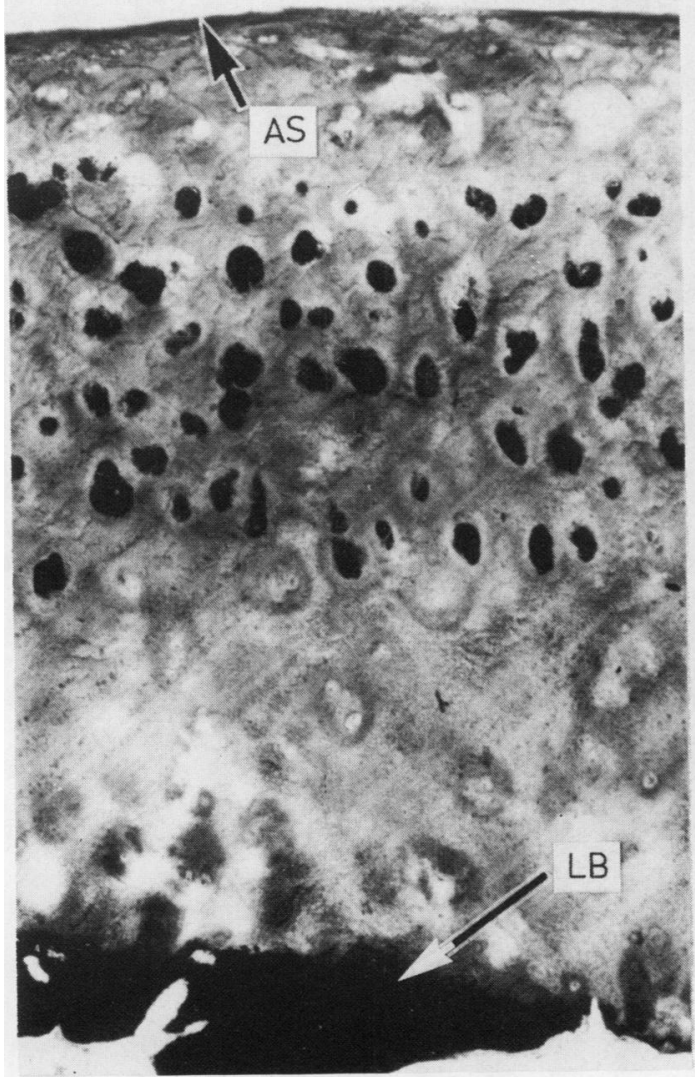

Fig. 9 Calcific deposits (black) around chondrocyte lacunae and as discrete masses in the intermediate layer of articular cartilage of the femoral head. AS is the articular surface and $L B$ is the subjacent lamellar bone.

Haematoxylin and eosin and von Kossa $\times 40$.

relative brightness densities of their images. Thus cartilage could be readily distinguished from lamellar bone. In the costal cartilage and in the articular cartilage of the femoral head bright deposits were seen around chondrocyte lacunae precisely corresponding to those sites which in the microscopic studies contained calcific deposits (Fig. 11). In the intervertebral discs larger more irregular bright deposits were observed. $X$-ray spectra were obtained from multiple areas of these deposits in the several specimens studied. From these spectra it was evident that phosphorus (P) and calcium (Ca) were present in the deposits. The $x$-ray emissions at each point were measured and the ratio of P:Ca calculated. The mean value of this ratio for the costal cartilage deposits was $\mathbf{0 . 8 8}$, for the intervertebral disc deposits 0.92 and for the femoral head deposits 0.98 (Fig. 12).

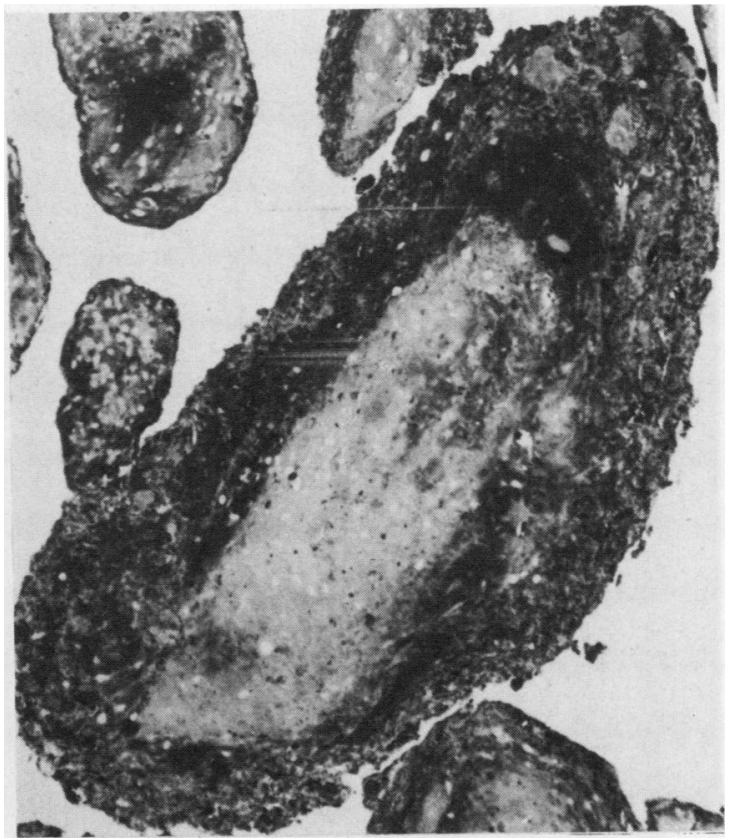

Fig. 10 Darkly staining calcific deposits mainly on the surface of the villi of the hip joint synovium. von Kossa $\times 40$.

\section{Discussion}

The presence of large quantities of homogentisic acid in the urine of this case indicates that the observed ochronosis was the consequence of alkaptonuria. The preparation of undecalcified thin sections allowed the demonstration of calcific deposits. These were present in the matrix of tracheal, costal, femoral and patellar cartilage both around chondrocyte lacunae and as discrete masses. Deposits were also present in both annular and nuclear zones of intervertebral discs. Positive reactions with alizarin red $S$ indicated the presence of calcium ions and positive reactions with the von Kossa technique indicated companion anions. Green-white fluorescence after the application of the morin technique was evidence that some of these anionic companions were in the form of pyrophosphate. ${ }^{12}$ This was further supported by weak positive birefringence with polarising light microscopy. ${ }^{13}$

Dieppe $e a^{14}$ have described the use of scanning electron microscopy (SEM) and $x$-ray microanalysis in the distinction of the several forms of calcium phosphate (calcium hydroxyapatite, calcium pyrophosphate dihydrate, calcium orthophosphate dihydrate and dicalcium phosphate dihydrate) which may occur in articular tissues. Crocker et $a^{15}$ have also described a correlative light microscopical and 


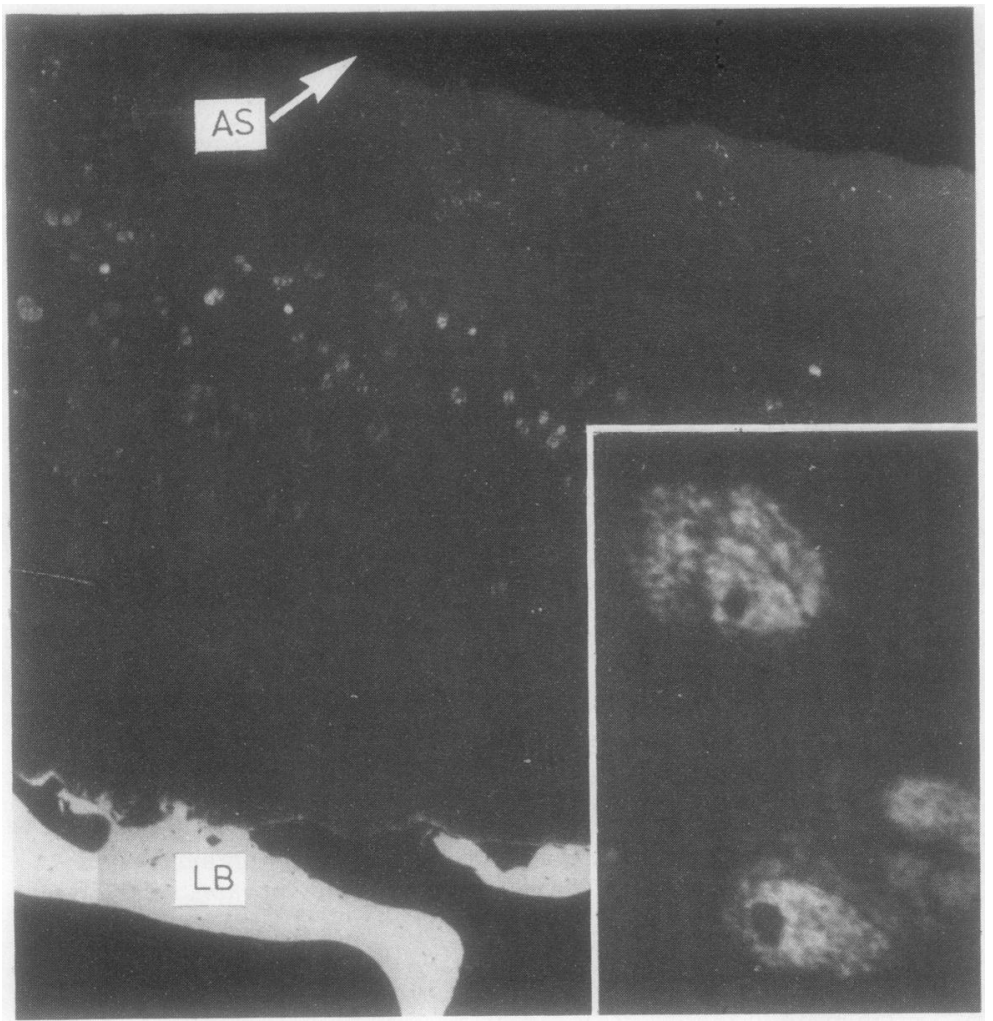

Fig. 11 Back scattered electron image of femoral head cartilage. The bright deposits in the intermediate zone correspond to the calcific deposits shown in Fig. 10. The inset is a higher magnification $(\times 600)$ showing the lacunar and perilacunar disposition of the deposits. AS is the articular surface and $L B$ the subjacent lamellar bone $\times 40$.

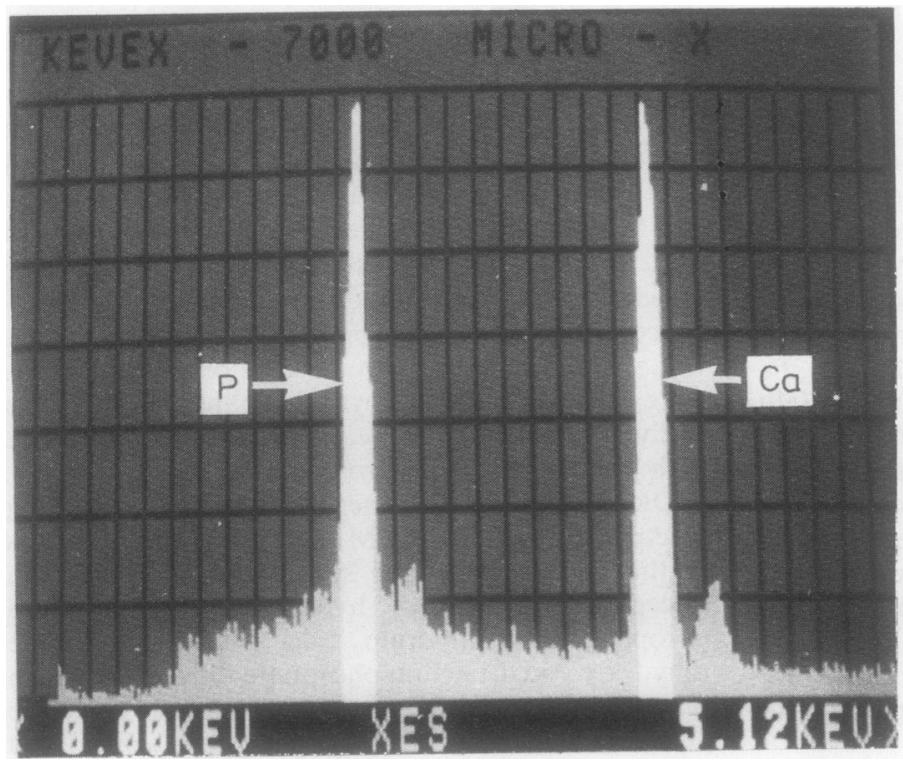

Fig. $12 x$-ray spectrum of a calcific deposit in the femoral head cartilage. Phosphorus $(P)$ and calcium $(\mathrm{Ca})$ are present. The P:Ca ratio is 0.98 . 
$x$-ray microanalytical method of identifying calcium pyrophosphate dihydrate (CPPD) crystals of differing morphology and calcium hydroxyapatite crystals in synovial fluid. These workers described a P:Ca ratio of $0.95 \pm 0.02$ for pure triclinic calcium pyrophosphate dihydrate and a value of $0.45 \pm 0.02$ for pure calcium hydroxyapatite. The P:Ca values obtained for the deposits studied in the present case are essentially similar to that of pure triclinic CPPD indicating that the majority of the observed calcific deposits is in the form of CPPD.

Calcific deposits were also present on the surface of synovial villi of the hip joint and these deposits also gave reactions indicating the presence of CPPD. There are three published examples ${ }^{9-11}$ of the convincing demonstration of CPPD in either synovial membrane or synovial fluid in large diarthrodial joints of patients with ochronotic arthropathy. In addition, Rynes et al $^{11}$ stated that their review of the histories and radiographs of five other patients with ochronosis revealed two cases of chrondrocalcinosis of knee joints and both patients had acute episodes of arthritis consistent with pseudogout (although CPPD was not specifically demonstrated). A fourth case of synovial CPPD deposition was documented by Bywaters $e t a l^{8}$ and this will be discussed in more detail later.

The presence of radio-opacities in the costal cartilages is a well recognised feature of ochronosis, but there have been no previous descriptions of the morphological basis of this phenomenon. It is apparent that there are not only intrachondral deposits of CPPD but also a process of endochondral ossification. Erosion of both unmineralised and mineralised matrix by multinucleated giant cells identical to chondroclasts (osteoclasts) was followed by the laying-down of osteoid and mineralised bone by osteoblasts on the walls of excavations. This coupled process is an exact replica of the cutting cone/ closing cone which is the basic unit of function in the ossifying epiphyseal plate and in the remodelling of mature cortical and trabecular bone. Macrophagelike cells behind the multinucleated giant cells contained intracytoplasmic ochronotic pigment granules thus raising the interesting possibility that these uninucleated cells were shed from the giant cells.

In the intervertebral discs pigment deposition was not uniform and CPPD deposits were not necessarily related to the focal ochronotic accumulations. Endochondral ossification with cutting cone/closing cone processes was also observed and it was again characterised by erosion of CPPD deposits by multinucleated cells with ossification of the walls of the excavations to provide osseous continuity with the bodies of the related vertebrae. The tissue in which this chondroblastic/osteoblastic differentia- tion occurred seemingly arose by protrusion of narrow tissue through defects in the limiting plates of the vertebral bodies. Obviously this endochondral ossification process resulted in bars of bone penetrating the discs and linking up of these caused partial or complete ankylosis. In the epiphyseal plate calcification of the matrix of hypertrophic cartilage is considered to induce cutting cone/closing cone activity which results in ossification. It would seem that deposits of CPPD have a similar inductive property.

Bywaters et $a l^{8}$ said that the characteristic radiological opacity of the intervertebral regions in ochronosis has been attributed to bony sclerosis following breakup and loss of cartilage. They further stated that histological and crystallographic studies of intervertebral disc material from a case had shown the presence of hydroxyapatite in the degenerating disc material. By contrast the synovial membrane contained CPPD crystals (shown by $x$-ray diffraction). The present study indicates that deposition of CPPD may occur in the intervertebral disc in ochronosis and this is associated with the formation of conventional trabecular bone of which hydroxyapatite is a component.

One interesting observation was that of replacement of one intervertebral disc by a cleft space bounded by hyperplastic bone. The surface features of this bone were identical to those of exposed subchondral bone in grade IV osteoarthrosis. It is interesting to speculate that movement occurred between these two surfaces.

In the articular cartilage of the hip and knee joints calcific deposits were present as discrete masses in the intermediate zone of sites with an intact surface. Bjelle $^{16}$ has illustrated similar deposits in pyrophosphate arthropathy not related to ochronosis and has pointed out that it is difficult to explain this peculiar localisation and has also pointed out that equivalent calcifications in animals consist of hydroxyapatite. ${ }^{17}$ In the present case calcific deposits were noted around chondrocyte lacunae (in tracheal, costal, femoral and patellar cartilage). This deposition implies that the chondrocytes have a role in the deposition of the calcium salts by mechanisms which can only be speculative. However, the deposition of calcific salts around the lacunae, once accomplished, has serious implications for the continued survival of the chondrocyte. In the hypertrophic cell zone of the epiphyseal plate the deposition of hydroxyapatite in the prelacunar matrix (by the activity of the chondrocyte) is considered to impair diffusion of nutrients and metabolites to and from the chondrocyte and to result thereby in the death of the cell. It is conceivable that a similar effect pertains in the present case and that this pericellular calcification also 
causes chondrocyte necrosis and initiates the arthropathic process.

Pyrophosphate is an important intermediary product of metabolism and is produced in large amounts in all tissues usually being rapidly broken down by pyrophosphatases. Increased synthesis, diminished enzymatic degradation, lowered solubility or a combination of these factors may be responsible for CPPD deposition. Thus in hypophosphatasia there is a deficiency of pyrophosphatase (alkaline phosphatase), a defect in breakdown of pyrophosphate and a pyrophosphate arthropathy. ${ }^{18}$ It is also of interest that ankylosis of the vertebral column has been observed in familial pyrophosphate arthropathy. ${ }^{16}$

The observations made in this case allow the formulation of the hypothesis that ochronotic arthropathy is, in part at least, a chronic pyrophosphate arthropathy and that the osseous organisation of CPPD deposits in the intervertebral disc provides a mechanism of partial or complete ankylosis of the vertebral column. Further, the perilacunar deposition of calcific material causes chondrocyte necrosis and initiates an osteoarthrotic process in the articular cartilage of large diarthrodial joints. It is not possible to make a definitive statement about the exact relation between alkaptonuria and CPPD deposition. It many be, however, that homogentisic acid excess so alters the milieu interieure that pyrophosphate excess also occurs.

\section{References}

${ }^{1}$ Virchow R. Ein Fall von allgemeiner Ochronose der Knorpel und knorpelahnlichen Theile. Virchows Arch [Pathol Anat] 1866;37:212-9.

${ }^{2}$ Harrold AJ. Alkaptonuric arthritis. J Bone Jt Surg 1956;38B:532-8.
${ }^{3}$ Laskar FH, Sargison KD. Ochronotic arthropathy. A review with four case reports. J Bone Jt Surg 1970;52B:653-66.

4 Oppenheimer BS, Kline BS. Ochronosis; with a study of an additional case. Arch Intern Med 1922;29:732-47.

${ }^{5}$ Lichtenstein L, Kaplan L. Hereditary ochronosis. Pathologic changes observed in two necropsied cases. Am J Pathol 1954;30:99-125.

- O'Brien WM, Banfield WG, Sokoloff L. Studies on the pathogenesis of ochronotic arthropathy. Arthritis Rheum $1961 ; 4: 137-52$.

' Hamilton EBD. Diseases associated with CPPD deposition disease. Arthritis Rheum 1976;19:353-7.

- Bywaters EGL, Dorling J, Sutor J. Ochronotic densification. Ann Rheum Dis 1970;29:563.

- Steiger U, Lagier R. Combined anatomical and radiological study of the hip joint in alcaptonuric arthropathy. Ann Rheum Dis 1972;31:369-73.

${ }^{10}$ Reginato AJ, Schumacher HR, Martinez VA. Ochronotic arthropathy with calcium pyrophosphate crystal deposition. Arthritis Rheum 1973;16:704-14.

"Rynes IR, Sosman JL, Holdsworth DE. Pseudogout in ochronosis. Report of a case. Arthritis Rheum 1975;18:21-5.

${ }^{12}$ Chaplin AJ. Calcium pyrophosphate. Histological characterisation of crystals in pseudogout. Arch Pathol Lab Med 1976;100:12-15.

${ }^{13}$ Currey HLF. Examination of joint fluids for crystals. Proc $R$ Soc Med 1968;61:909-71.

14 Dieppe PA, Huskinson EC, Crocker P, Willoughby DA. Apatite deposition disease. A new arthropathy. Lancet 1976;i:266-8.

is Crocker PR, Dieppe PA, Tyler CT, Chapman SK, Willoughby DA. The identification of particulate matter in biological tissues and fluids. J Pathol 1977;121:37-40.

${ }^{16}$ Bjelle A. Pyrophosphate arthropathy. Scand J Rheumatol 1979;8:145-53.

${ }^{17}$ Sokoloff L, Jay GE Jr. Natural history of degenerative joint disease in small laboratory animals. 4. Degenerative joint disease in the laboratory rat. Arch Pathol 1956;62:140-2.

${ }^{18}$ Eade AWJ, Swannell AJ, Williamson N. Pyrophosphate arthropathy in hypophosphatasia. Ann Rheum Dis 1981; 40:164-70.

Requests for reprints to: Dr J McClure, Department of Histopathology, University Hospital of South Manchester, Nell Lane, Withington, Manchester M20 8LR. 